\title{
ESTUDO DAS ALTERAÇÕES ULTRA-ESTRUTURAIS EM PLEURA DE RATOS PÓS PLEURODESE POR TALCO
}

\author{
RAT PLEURAL ULTRA STRUCTURAL CHANGES AFTER TALC PLEURODESIS
}

Tiago J. Refosco ${ }^{1}$ Isabela Dutra ${ }^{1}$; Airton Schneider, TCBC-RS ${ }^{2}$

\begin{abstract}
RESUMO: Objetivo: O talco é considerado um agente efetivo de pleurodese freqüentemente utilizado no tratamento de derrame pleural maligno. Entretanto, questiona-se sua segurança em relação à carcinogênese. O objetivo deste trabalho é descrever as alterações ultra-estruturais encontradas nas pleuras de ratos submetidos à pleurodese com talco buscando esclarecer sua segurança em relação à carcinogênese. Método: Neste estudo randomizado foram utilizados seis ratos machos do tipo Wistar, pesando $250 \mathrm{~g}$. Através de uma toracotomia mínima no quinto espaço intercostal esquerdo foi colocado lavado de talco em concentrações de $0,5 \mathrm{mg}$ $(\mathrm{n}=2)$ e $0,1 \mathrm{mg}(\mathrm{n}=2)$ de talco por grama de peso. Dois ratos foram apenas anestesiados e foi instilado solução fisiológica $(\mathrm{NaCl} 0,9 \%)$ intrapleural para configurar o grupo controle. Os ratos foram mortos após 45 dias. As alterações morfológicas e morfométricas ultra-estruturais foram analisadas através de microscopia eletrônica de transmissão. Resultados: A análise ultra-estrutural das pleuras submetidas à talcagem demonstrou desorganização celular do tecido conjuntivo, evidenciando alterações nucleares, mitocondriais e espessamento da camada submesotelial. O grupo controle manteve a arquitetura celular preservada. Conclusões: Este estudo revelou alterações da estrutura celular da pleura exposta ao talco quando comparado ao grupo controle. (Rev. Col. Bras. Cir. 2004; 31(6): 345-348).
\end{abstract}

Descritores: Pleurodese; Pleura; Carcinogênese; Talco; Ratos.

\section{INTRODUÇÃO}

O talco foi descrito, pela primeira vez, como agente efetivo de pleurodese por Bethune ${ }^{1}$ em 1935, cujos estudos foram posteriormente confirmados em modelos animais por inúmeros pesquisadores ${ }^{2-4}$. Essa substância vem-se mostrando claramente superior a outras, como tetraciclina e bleomicina, quanto à efetividade da pleurodese $e^{4}$.

Pleurodese por instilação de substâncias na cavidade pleural é o procedimento mais comumente utilizado como tratamento de derrame pleural recidivante maligno, embora possa também ser usado em derrames benignos, pneumotórax recorrente, quilotórax e empiema.

$\mathrm{O}$ uso do talco como agente promotor de pleurodese não é, entretanto, livre de efeitos adversos. Kennedy et al ${ }^{5}$ demonstraram que febre, dor de intensidade variável, empiema e falência respiratória podem ocorrer, principalmente ao se utilizarem doses altas de talco. Werebe et al ${ }^{6}$ demonstraram rápida absorção das partículas de talco pela pleura, sendo que cristais dessa substância foram encontrados fora da cavidade pleural 24 horas após o experimento. Em estudo subseqüente os mesmos autores relataram a absorção sistêmica e depósitos de cristais em vários órgãos de ratos submetidos à pleurodese com talco.

Considerando a ampla utilização do talco como um agente eficaz na indução de pleurodese, objetivamos, através deste trabalho, descrever as alterações ultra-estruturais nas pleuras afetadas pelo talco.

Não há evidências de risco de malignidade com o uso de talco. A presença de alterações ultra-estruturais precursoras de neoplasia em pleura de ratos expostos ao talco recomendaria que as indicações da talcagem fossem exclusivas para doenças malignas.

\section{MÉTODO}

Foram utilizados seis ratos machos do tipo Wistar pesando aproximadamente $250 \mathrm{~g}$. Os ratos foram divididos em dois grupos de dois animais cada, de acordo com a dose de talco recebida.

Os animais foram tratados segundo normas do "Guide for Care and Use of Animals" (NIH publication 85-23, revised 1985) e de acordo com a lei 6638 de 08 de maio de 1979, além do Manual para técnicas de biotério, que regulamenta cuidados mínimos e dignos para animais de experimentação tendo orientações preconizadas pelo Colégio Brasileiro de Experimentação Animal (COBEA). O projeto foi aprovado pelo comitê de ética da UFRGS.

A anestesia dos animais foi feita com Pentobarbital Sódico na dose de $50 \mathrm{mg} / \mathrm{kg}$ via intraperitoneal complementada com lidocaína $2 \%$ sem vasoconstrictor no local da incisão.

O procedimento foi realizado através de toracotomia mínima no quinto espaço intercostal esquerdo e colocação de lavado de talco por meio de uma sonda de aspiração fina (Sondaplast-Mat.Medo.Mat.Hospi. * ${ }^{\circ} 8$ ) com concentrações de $0,5 \mathrm{mg} / \mathrm{g}$ e $0,1 \mathrm{mg} / \mathrm{g}$ de talco por grama de peso. Dois ratos foram apenas anestesiados e foi instilado solução fisiológica $(\mathrm{NaCl} 0,9 \%)$ intrapleural para configurar o grupo controle.

Foi utilizado talco livre de asbesto, esterilizado por autoclave, sendo que uma amostra utilizada para talcagem foi

1. Acadêmicos de Medicina da ULBRA

2. Professor Adjunto do Departamento de Ciências Morfológicas, ICBS, UFRGS; Prof Adjunto de Cirurgia Torácica da ULBRA.

Recebido em 19/04/2004

Aceito para publicação em 03/08/2004

Trabalho Desenvolvido no Laboratório de Pesquisa - ICBS - UFRGS e no Centro de Microscopia Eletrônica da UFRGS. 
semeada em placas contendo agar para cultivo de bactérias sendo que todas as doses foram diluídas em $2 \mathrm{ml}$ de água destilada.

Após o procedimento o pneumotórax residual foi aspirado pela mesma cânula e a parede torácica fechada com fio de nylon monofilamentar.

Os ratos foram mortos por decapitação 45 dias após a realização da cirurgia,com exposição da cavidade pleural para retirada de fragmentos de pleura.

$\mathrm{O}$ estudo das alterações morfológicas e morfométricas ultra-estruturais foi executado através de microscopia eletrônica de transmissão, utilizando o microscópio eletrônico JEOL jem-1200 ex II da Universidade Federal do Rio Grande do Sul.

O material da biópsia foi imediatamente colocado em solução fixadora com glutaraldeído $25 \%$, paraformaldeído $2 \%$, tampão fosfato $0,12 \mathrm{M}$. As amostras dos seis ratos foram subdivididas em cinco subamostras de cada.

As amostras foram então lavadas em tampão fosfato 0,1 M (três banhos de 30 minutos), seguido pelo tetróxido de ósmio $2 \%$ com tampão $0,2 \mathrm{M}$, para uma pós-fixação de 30 minutos.

A desidratação processa-se na série: acetona ou etanol 30\%, 50\%, 70\%, 95\%(dois banhos) e 100\%(dois banhos). Todas as passagens têm duração de 10 minutos, com exceção das duas últimas que tem duração de mais 20 minutos.

A pré-embebição dos materiais é feita em uma mistura de epon 812 com acetona pura nas seguintes proporções:

a) Uma parte de epon + duas partes de acetona por 2 horas;

b) Uma parte de epon + uma parte de acetona por 24 horas;

c) Duas partes de epon + uma parte de acetona por 2 horas;

* Todo o processo de pré-embebição é feita no Rotator.

Mistura da Resina Epon:

Epon 812 .................................. 25ml;

MNA …...................................... 17ml;

DDSA …................................. 08ml;

DMP-30 .................................. 32 gotas.

A embebição é feita com epon 812 puro durante 24 horas.

Para a inclusão são usados moldes siliconados chatos (Embedding molds Sigma) para que as amostras fossem então orientadas.

A polimerização é efetuada em estufa $60^{\circ} \mathrm{C}$ constantes por 72 horas.

Os cortes semifinos foram feitos no ultramicrótomo, Leica Ultracut UCT, sendo utilizada navalha de vidro, com uma espessura de $800 \mathrm{~nm}$ e corados com solução aquosa de azul de toluidina $1 \%$.

Os cortes ultrafinos foram confeccionados com uma espessura de $70 \mathrm{~nm}$ no mesmo ultramicrótomo, com navalha de diamante. Para o contraste dos cortes ultrafinos utilizou-se solução aquosa de acetato de uranila $2 \%$ e, após, citrato de chumbo.
Tabela 1 - Resumo do método de estudo

\begin{tabular}{llll}
\hline & Grupo1 & Grupo2 & Controle \\
\hline N $^{\text {o de Ratos }}$ & 2 & 2 & 2 \\
Peso em g. & $200-300$ & $200-300$ & $200-300$ \\
Dose de Talco & $0,5 \mathrm{mg} / \mathrm{g}$ & $0,1 \mathrm{mg} / \mathrm{g}$ & $\mathrm{N}$ \\
Tempo de seguimento & $45 \mathrm{dias}$ & $45 \mathrm{dias}$ & $45 \mathrm{dias}$ \\
Análise por mic. Elet. & 2 & 2 & 2 \\
\hline
\end{tabular}

Os cortes ultrafinos foram colocados em telas de 200 mesh G200-Cu, e posteriormente analisados no microscópio Eletrônico de transmissão do CME/UFRGS.

A Tabela 1 resume o método do estudo.

Para avaliar os resultados, do ponto de vista estatístico, utilizou-se o teste não-paramétrico qui-quadrado, considerando $\mathrm{p}<0,05$ como significância estatística.

\section{RESULTADOS}

Na pleura normal observou-se o mesotélio formado por um epitélio pavimentoso simples com uma única camada de células, todas com suas membranas celulares intactas, bem delimitadas e com alguns prolongamentos. A maior parte delas apresentou, intracitoplasmaticamente, grânulos elétron-densos configurando diversos vacúolos com densidade semelhante à proteína polissacarídica, além de uma grande quantidade de retículo endoplasmático liso e mitocôndrias de tamanho normal, com cristas e matriz bem visíveis.O núcleo celular demonstrou heterocromatina bem delimitada, tendo uma proporção adequada em relação à eucromatina. Profundamente às células epiteliais evidenciou-se a lâmina basal do tecido epitelial com sua espessura e formatos preservados apoiados sobre fibras reticulares e colágenas, além de inúmeros fibroblastos. Em meio ao tecido colágeno também encontrou-se vacúolos com grânulos elétron-densos em abundância.

A Figura 1 demonstra a integridade da pleura submetida à instilação de soro fisiológico. Na Figura 2 pode-se observar a presença de grânulos elétron-densos.

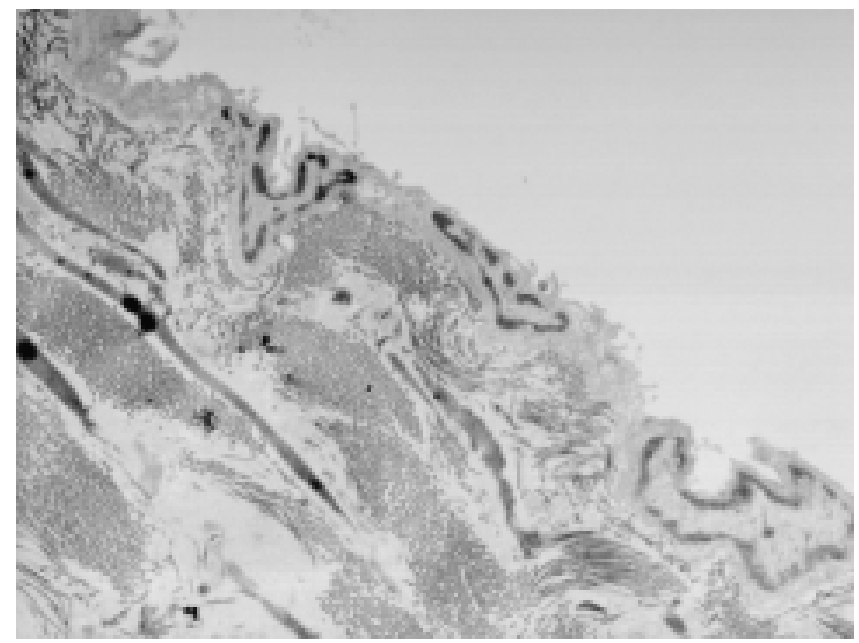

Figura 1 - Pleura normal: rato submetido à instilação de soro fisiológico. 


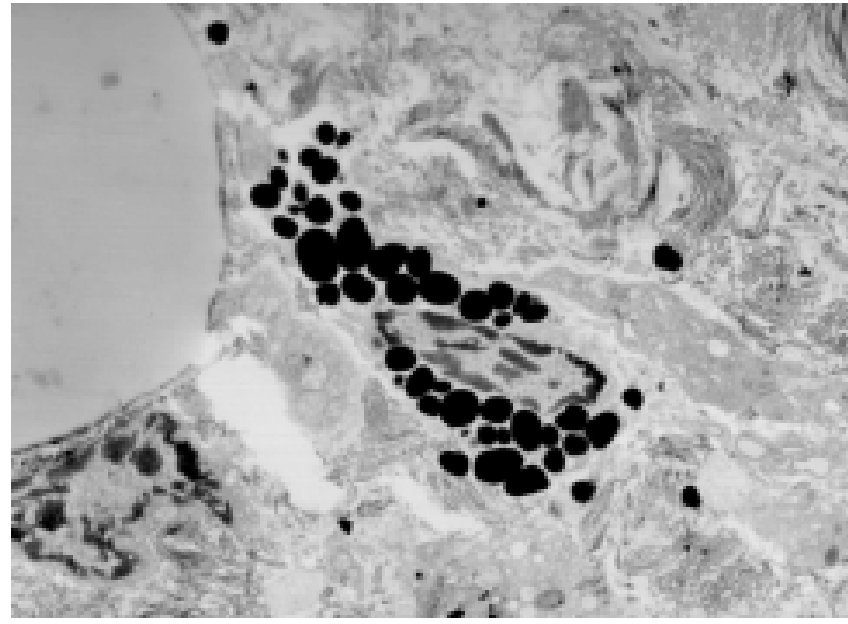

Figura 2 - Pleura normal com grânulos elétron-densos.

Entretanto, nas pleuras dos ratos submetidos ao talco, observou-se células com um intenso depósito de cristais semelhantes ao carbonato de cálcio do talco depositado sobre a superfície mesotelial. Diversas dessas partículas foram encontradas intracitoplasmaticamente cercadas por grânulos lisossômicos. As paredes celulares dessas células demonstramse mais irregulares e suas ligações com outras membranas celulares não parecem mais tão justapostas. Os núcleos apresentam uma desorganização com aumento da eucromatina em relação à heterocromatina. Em todas as amostras a lâmina basal do tecido mesotelial se demonstrou mais delgada e menos regular. O tecido conjuntivo abaixo apresentou uma grande proliferação com aumento do número de fibroblastos e certa desorganização estrutural das fibras colágenas. Também nestas células podemos identificar a presença de cristais intracitoplasmáticos sem presença de fagossomos, com proliferação de complexos de Golgi e alterações como aumento do tamanho das mitocôndrias e alteração de sua matriz. O tecido muscular da parede torácica adjacente à pleura parietal não demonstrou qualquer alteração estrutural nas nossas amostras.

As Figuras 3 e 4 demonstram as alterações ultra-estruturais quanto à morfometria e morfologia da pleura submetida à exposição ao talco.

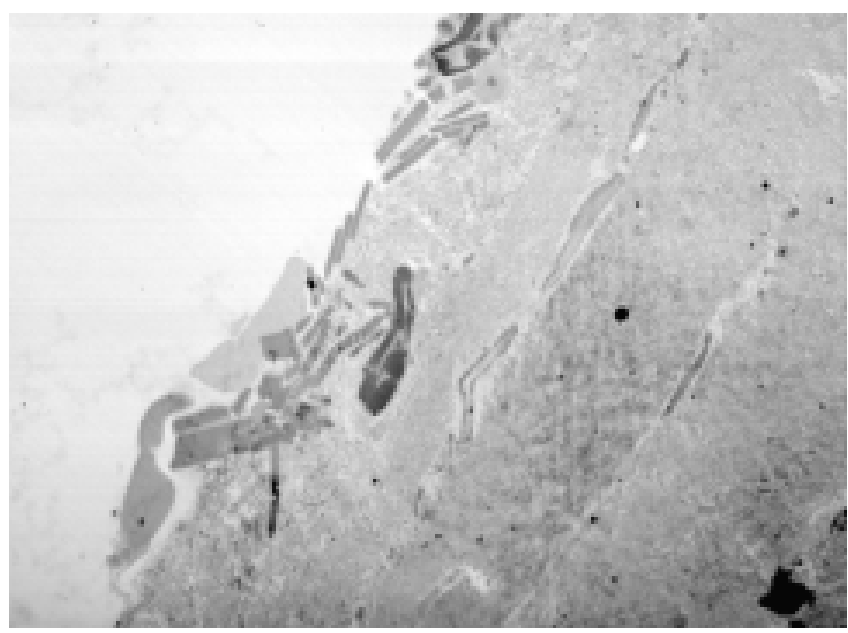

Figura 3 - Alterações encontradas na pleura dos ratos submetidos à talcagem na dose de $0,5 \mathrm{mg} / \mathrm{g}$.
Embora o número de ratos seja pequeno, com o método do qui quadrado estas alterações mostraram-se estatisticamente significativas $(\mathrm{p}<0,05)$ quando comparadas às do grupo controle.

Entretanto, não houve diferença nos achados entre as pleuras submetidas à diferentes concentrações de talco $(\mathrm{p}>0,05)$.

\section{DISCUSSÃO}

Ainda não se conhece o mecanismo exato pelo qual o talco induz à pleurodese. A instilação dessa substância na cavidade pleural visa, através de reação inflamatória, produzir sínfise fibrosa das pleuras parietal e visceral ${ }^{7}$.

A talcagem apresenta maior ação fibrosa na cavidade pleural quando comparado à abrasão mecânica ${ }^{8}$, mostrandose também superior ao Corynebacterium parvum, bleomicina, tetraciclina ${ }^{4}$, nitrato de prata, doxiciclina e minociclina atomizada ${ }^{6,9}$. Xie et $a^{4}$ demonstraram que o derrame exsudativo que se forma após a talcagem resolve-se dentro de quatro dias e, assim que isso ocorre, as adesões entre as pleuras tendem a aumentar, enquanto a reação inflamatória pleural tende a diminuir. Kennedy et al ${ }^{9}$, ao analisar os achados histológicos e o líquido pleural pós-talcagem, relataram desnudamento do mesotélio em 24 horas, predominância de neutrófilos no líquido pleural, espessamento progressivo da pleura em sete dias (com aderências de fibrina em áreas de desnudamento mesotelial e de dano à membrana basal), além de resolução lenta da inflamação pleural com predomínio de mononucleados enquanto havia deposição de colágeno e proliferação de fibroblastos. Van der Heuvel et a ${ }^{10}$ relatam que, após a administração de talco na cavidade pleural para indução de pleurodese em pacientes com pneumotórax espontâneo ou derrame pleural maligno, ocorre rápido influxo de neutrófilos polimorfonucleares, seguido de um acúmulo de macrófagos no líquido pleural. Ainda, há aumento da produção de interleucina 8 (IL-8) e proteína quimiotáxica para monócitos (MCP-1), o que justifica a presença de neutrófilos e o acúmulo de monócitos no processo inflamatório induzido pelo talco no espaço pleural .

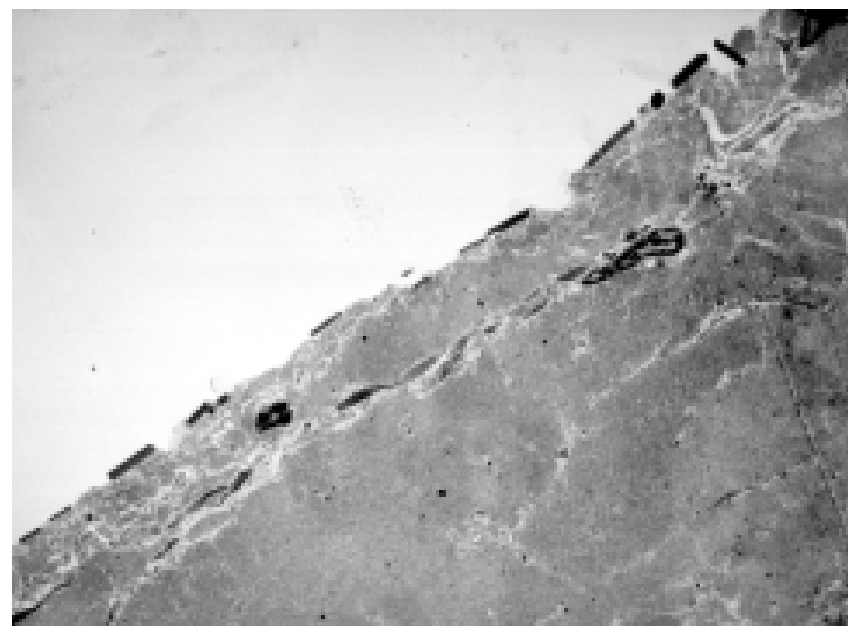

Figura 4 - Pleura dos ratos submetidos à talcagem na dose de $0,1 \mathrm{mg} / \mathrm{g}$. 
O significado clínico dos achados descritos neste trabalho é algo extremamente novo e, portanto, de difícil interpretação.

A presença de proliferação da porção da eucromatina nuclear associada às inclusões citoplasmáticas de partículas sugere um aumento da atividade nuclear, podendo inferir tanto um descontrole da divisão nuclear bem como alterações de defesa celular. Esta alterações são descritas em tumores em fase inicial ${ }^{11}$.

A proliferação do tecido colágeno adjacente ao mesotélio mostra que há uma forte reação de corpo estranho levando a uma defesa característica, porém até onde essa desestruturação do colágeno com proliferação de fibroblastos e inclusão de alguns macrófagos é inocente, é algo em aberto.

Vargas $e t a l^{3}$ demonstraram que a capacidade dos fibroblastos em secretar colágeno bem como a atividade das metaloproteinases (enzimas que degradam colágeno) são moduladas pelo uso de corticóides, não havendo adesão pleural com o uso concomitante dos mesmos.

O nosso trabalho reaviva a discussão que, embora o talco seja um excelente agente esclerosante, talvez o mais eficaz, sua utilização em patologias benignas deva ser reavaliada e estudada mais profundamente.

Concluindo, foram evidentes as alterações ultra-estruturais encontradas nas pleuras afetadas pelo talco em comparação ao grupo controle. A talcagem ainda é o método mais efetivo de se obter adesão pleural, porém sua segurança com relação à carcinogênese não está estabelecida. Fazem-se necessários estudos subseqüentes para que essas alterações tenham confirmação de seu significado clínico.

\section{AGRADECIMENTOS}

Este trabalho não seria possível de ser realizado sem o auxilio dos profissionais do Centro de Microscopia Eletrônica da UFRGS.

\begin{abstract}
Background: Talc is considered an effective agent for pleurodesis often used in the treatment of malignant pleural effusion. However, its safety related to carcinogenesis is questioned. The objectives are to describe ultra-structural alterations detected in pleurae of rats submitted to talc pleurodesis aiming at assessing its safety in relation to carcinogenesis. Methods: In this randomized study, six Wistar rats weighing $250 \mathrm{~g}$ were used. Through a minimal thoracotomy into the fifth left interspace, it was inserted talc in $0.5 \mathrm{mg}$ concentrations $(n=2)$ and $0.1 \mathrm{mg}(n=2)$ each gram weight. Two rats were only anesthetized and a physiological solution ( $\mathrm{NaCl} 0.9 \%$ )was intrapleurally inserted to represent the control group. The rats were killed after 45 days. Ultra-structural morphological and morphometric alterations were analyzed by transmission electronic microscopy. Results: Ultra-structural analysis of pleurae submitted to talc has shown cellular disorganization of the conjunctive tissue evidencing nuclear, mitochondrial alterations and submesothelial spacing. The control group has maintained the cellular architecture preserved. Conclusions: This study revealed alterations in the cellular structure of talc-exposed pleura when compared to the control group.
\end{abstract}

Key words: Pleurodesis; Pleura; Oncogenesis; Talc; Rats.

\section{REFERÊNCIAS}

1. Bethune N - Pleural poudrage: new techniques for deliberate production of pleural adhesions as preliminary to lobectomy. $\mathbf{J}$ Thorac Surg, 1935, 4(1):251-261

2. Mitchem RE, Herndon BL, Fiorella RM, et al. - Pleurodesis by autologous blood, doxycycline, and talc in a rabbit model. Ann Thorac Surg, 1999, 67(4):917-921.

3. Vargas FS, Teixeira LR, Vaz MA, et al. - Silver nitrate is superior to talc slurry in producing pleurodesis in rabbits. Chest, 2000, 118(3):808-813.

4. Xie C, McGovern JP, Wu W, et al. - Comparisons of pleurodesis induced by talc with or without thymol iodide in rabbits. Chest, 1998, 113(3):795-799.

5. Kennedy L, Rusch VW, Strange C, et al. - Pleurodesis using talc slurry. Chest, 1994, 106(2):342-346.

6. Werebe EC, Pazetti R, Milanez de Campos JR, et al. - Systemic distribution of talc after intrapleural administration in rats. Chest, 1999, 115(1):190-193.

7. Zimmer PW, Hill M, Casey K, et al. - Prospective randomized trial of talc slurry versus bleomycin in pleurodesis for symptomatic malignant pleural effusions. Chest, 1997, 112(2):430-434.
8. Whitlow CB, Craig R, Brady K, et al. - Thoracoscopic pleurodesis with minocycline versus talc in the porcine model. Surg Endosc, 1996, 10(11):1057-1059.

9. Kennedy L, Harley RA, Sahn SA, et al. - Talc slurry pleurodesis. Pleural fluid and histologic analysis. Chest, 1995, 107(6):17071712.

10. Van Der Heuvel MM, Smit HJ, Barbierato SB, et al. - Talcinduced inflammation in the pleural cavity. Eur Respir J, 1998, 12(6):1419-1423.

11. Heidelberg C-Chemical carcinogenesis, chemotherapy cancer's continuing core challenges-G.H.A. Clowes Memorial Lecture. Cancer Res, 1970, 30(6):1549-1569.

Endereço para correspondência:

Airton Schneider

Rua Cel. Bordini 896/401

90140-003 - Porto Alegre-RS

E-mail: airtonsc@terra.com.br 